\title{
An Improved Algorithm Research of Transit Signal Priority Based on Bi- objective Optimization Model
}

\author{
Tianhong Gu \\ Dept of Mechanical Engineering \\ Dalian Institute of Science and Technology \\ Dalian, China \\ e-mail: 125511616@qq.com
}

\author{
Huijian Cao \\ Transportation Institute \\ Shijiazhuang Tiedao University \\ Shijiazhuang, China \\ e-mail: 5780518@163.com
}

\begin{abstract}
Optimization of adaptive traffic signal timing is one of the most complex problems in traffic control systems. In order to obtain control parameters of bus signal priority of the urban roads, a bi-objective optimization model for signal timing is established based on real-time data of GPS. As the goal of optimization, the delay and stops are calculated respectively. And the corresponding constraint condition is built. The method can optimize green splits and offset at urban arterial road. A VISSIM simulation testbed was developed to evaluate the performance of the proposed bi-objective optimization model. The results show that the optimizer can produce TSP timing plans that benefit the transit vehicles while minimizing the impact of TSP on the general vehicles.
\end{abstract}

Keywords-Transit Signal Priority;Urban Arterial Road;Biobjective Optimization

\section{INTRODUCTION}

The importance of public transportation in providing sustainable mobility is being recognized by researchers and transportation authorities. Transit signal priority (TSP) has shown promise in improving the performance of inservice transit vehicles by reducing delay time.

Early Green and Extended Green are the two most commonly applied operational strategies in TSP systems. They both rely on shortening the green time of the opposing approaches to obtain extra green time for the prioritized phases. However it might have negative impact on other vehicles. How to reduce total vehicle queue delay and stops including the prioritized and non-prioritized phases has been the focus of study. Guangwei Zhou presents an adaptive transit signal priority (TSP) strategy that applies the parallel genetic algorithm (PGA) to optimize adaptive traffic signal control in the presence of TSP [1]. Ma Wajing proposed a new bus priority approach with coordinated signalized intersection group. Minimization of travel delay deviation at intersection group is as the control objective [2].

In the above theories, the number of stops is rarely considered. When the traffic capacity of non- prioritized phases is lower, the stops will increase sharply. Meanwhile it will cause environment pollution and the excessive consumption of energy. In this paper, a biobjective optimization mode was proposed based on the both objectives of delay and stops. And a calculation model of bus delay was proposed by using the characteristics of GPS real-time monitoring data. Based on it, the optimized signal timing at the intersections is obtained. A simulation study is then established and illustrates its effectiveness.

\section{ANALYTICAL FUNDAMENTALS OF OPTIMIZATION OBJECTIVE}

\section{A. Calculating bus motion trail by GPS technology}

Global Positioning System (GPS) collects accurate information on position and velocity of bus. Fig. 1 shows graphically the movement track of bus.

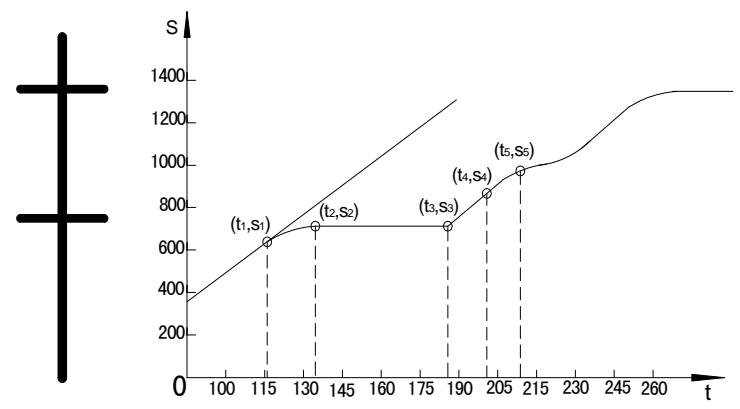

Figure 1. Bus motion trail

The distance $L$ between two intersections is assumed to be invariable. Furthermore assume that the buses move at a constant rate. The time $T_{b}$ when the bus reaches the stopping line is given by the following formula:

$$
\begin{aligned}
& \frac{d s}{d t}=v \\
& T_{b}=\frac{L-l_{2}}{v}+t_{2}-t_{1}
\end{aligned}
$$

Where,

$t_{1}=$ the time when the bus reaches the first intersection;

$t_{2}=$ the later time of GPS data;

$l_{2}=$ the bus position to the time $t_{2}$;

$v=$ running speed of bus.

B. Analytical fundamentals of delay on prioritized and non-prioritized phases

Traffic condition of intersection can be divided by saturation. Saturation is the ratio of vehicle number and 
Lane capacity in a cycle. Then the degree of saturation $X$ of an approach is:

$$
x=\frac{q \cdot C}{s \cdot g}
$$

Where,

$q$ =arriving rate of an approach;

$c$ =cycle length;

$g$ =the effective green light time;

$S=$ the capacity of an approach.

$x<1$ is an under saturated case when the approach arrivals do not exceed the capacity. As shown in Fig.2, assuming that waiting vehicle's numbers of $k$ cycle in signal intersection entrance lanes is $n(k-1)$. The effective green light time, the effective red light time and amber time respectively are $g(k), r(k)$ and $y(k)$. Cycle length of $k$ period is expressed by $c(k)$.According to delay $D(k)$ for a lane group is given by the following formula:

$$
D(k)=\frac{[r(k) \cdot s+n(k-1)][r(k) \cdot q(k)+n(k-1)]}{2(s-q(k))}
$$

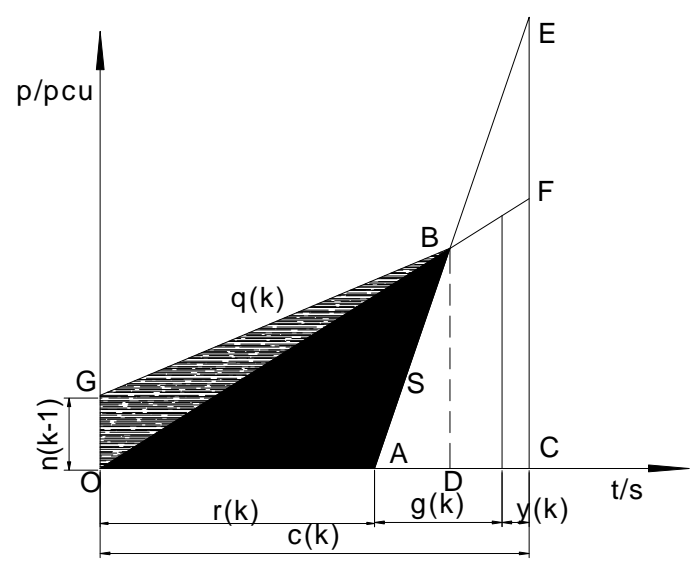

Figure 2. The calculation of vehicle delay

Consider the diagram shown in Fig. 3.If the approach is assumed to be under saturated and waiting vehicle's numbers of $k-1$ cycle is zero. Vehicle arrival rate $q(k)$ is a expression of segmented function which is

$$
q(k)=\left\{\begin{array}{cc}
q_{1}(x, k) & 0 \leq x \leq O B \\
q_{2}(x, k) & O B<x \leq B C \\
q_{3}(x, k) & B C<x<C D
\end{array}\right.
$$

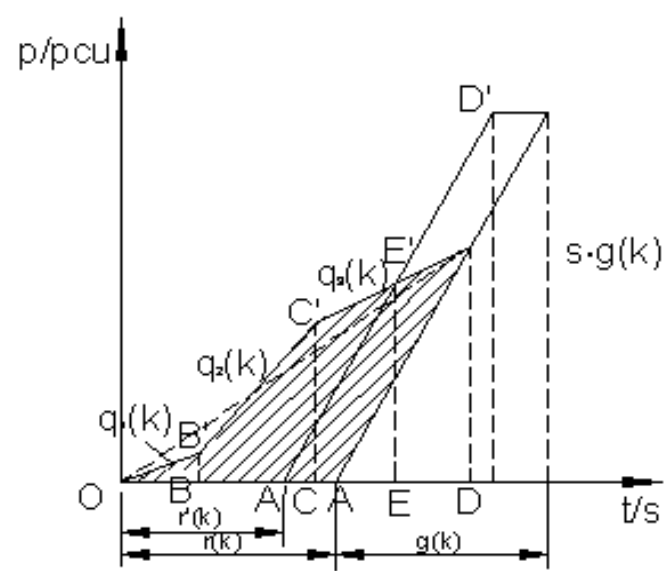

Figure 3. The calculation of vehicle delay when vehicle arrival rate is nonconstant

When Early Green is not operational, the vehicle delay $D(k)$ is summarized:

$$
\begin{aligned}
& D(k)=\int_{O}^{D} q(x, k) d x-\int_{A}^{D} S(x) d x \\
& =\int_{O}^{B} q_{1}(x, k) d x+\int_{B}^{C} q_{2}(x, k) d x+\int_{C}^{D} q_{3}(x, k) d x-\int_{A}^{D} S(x) d x
\end{aligned}
$$

When Early Green is not operational, the vehicle delay $D^{\prime}(k)$ is summarized:

$$
\begin{aligned}
& D^{\prime}(k)=\int_{O^{2}}^{E} q(x, k) d x-\int_{A^{\prime}}^{E} S(x) d x \\
& =\int_{O^{\prime}}^{B} q_{1}(x, k) d x+\int_{B}^{C} q_{2}(x, k) d x+\int_{C^{2}}^{E} q_{3}(x, k) d x-\int_{A^{\prime}}^{E} S(x) d x
\end{aligned}
$$

\section{Analytical fundamentals of delay on prioritized and non-prioritized phases}

The time and numbers is not constant when the vehicle reaches the stopping line. So during each signal cycle some vehicles are stopped before the stopping line. The speed of vehicles reduced from the driving normal to zero. This creates the phenomenon of parking.

According to the HCM the stopping rate, $h_{s}$, for a lane group is given by the following formula:

$$
\begin{gathered}
h_{s}=f \cdot\left(\frac{1-\lambda}{1-y}+\frac{N_{s}}{q C}\right) \\
N_{s}=\frac{e^{k}}{2(1-x)}
\end{gathered}
$$

Where,

$$
\begin{aligned}
& e=2.718 ; \\
& k=-1.33 \sqrt{s \cdot q} \cdot(1-x) / x \\
& N_{s}=\text { average queue length in unsaturated }
\end{aligned}
$$
intersection;

$f=$ Correction parameters.

\section{The influence of offset to bus delay}

With communication technology developing precisely calculating the delay time of buses can be achieved. In this 
paper, the change rate of movement track of bus is studied under the condition of new communication technology. The bus delay is calculated as:

$$
I_{b}=\left\{\begin{array}{cc}
x+T-T_{b} & 0 \leq x<T_{b}-g \\
0 & T_{b}-g \leq x<T_{b} \\
x-T_{b} & T_{b} \leq x<T
\end{array}\right.
$$

where, $I_{b}=$ the bus delay;

$x=$ offset between two intersection;

$T$ =cycle length;

$g=$ Green effective time.

\section{APPLICATION ANALYSIS}

\section{A. Basic data analysis}

As is shown in Fig.4, two typical adjacent two-phase signalized intersections were used to obtain estimates of the impact that Bi-objective Optimization Model have on the cross-street delays. Signal priority applies to the coordinated phases 2 and 6 . The impacts were evaluated for several bus flow (veh/h) and cross-street green time. The cross-street for this initial scenario had a flow ratio $(\mathrm{v} / \mathrm{s})$ of 0.30 which is indicative of an intersection operating well. As shown in Table1, all one needs is the signal timing plan and the traffic demand.

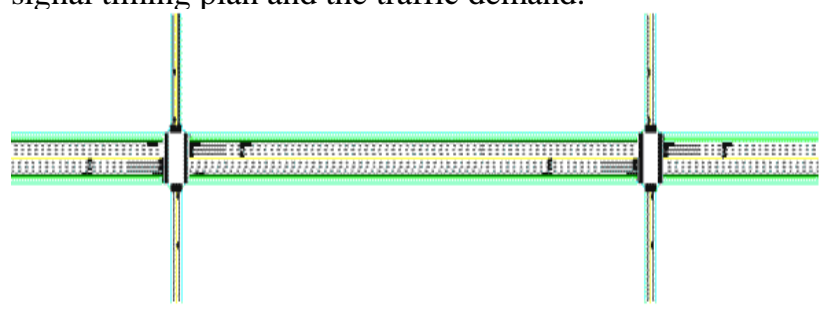

Figure 4. Two typical adjacent two-phase signalized intersections

TABLE I. SIGNAL TIMING FOR TWO TYPICAL ADJACENT INTERSECTIONS

\begin{tabular}{|c|c|c|c|c|c|c|}
\hline Phases & $\begin{array}{l}\text { Move } \\
\text { ments }\end{array}$ & $\begin{array}{c}\text { S } \\
\text { (veh/h) }\end{array}$ & $\begin{array}{c}\text { Green } \\
\text { time } \\
\text { (s) }\end{array}$ & $\begin{array}{c}\text { Cycle } \\
\text { (s) }\end{array}$ & $\begin{array}{c}\text { Lost } \\
\text { time } \\
\text { (s) }\end{array}$ & $\begin{array}{l}\text { flow } \\
\text { ratio } \\
(\mathrm{v} / \mathrm{s}) \\
\end{array}$ \\
\hline \multirow{3}{*}{$1(4,8)$} & straight & \multirow{3}{*}{1800} & \multirow{3}{*}{80} & \multirow{6}{*}{150} & \multirow{6}{*}{4} & \multirow{6}{*}{$<0.3$} \\
\hline & left & & & & & \\
\hline & right & & & & & \\
\hline \multirow{3}{*}{$2(2,6)$} & straight & \multirow{3}{*}{1800} & \multirow{3}{*}{66} & & & \\
\hline & left & & & & & \\
\hline & right & & & & & \\
\hline
\end{tabular}

\section{B. The application of Bi-objective Optimization} Model

Aimed at the defect of signal timing to single objective optimization, the bi-objective optimization model was proposed and adopted. Based on delay time and stops, the generalized bi-objective function was set up according to multi-objective optimization theory. The variable number is reduced in this algorithm and algorithm efficiency is improved. The model is as follows: $\min f_{1}\left(g(k), x_{o s}\right)=\sum_{i=1}^{2} D_{i}(k)+\bar{d}_{q_{i}}(k) \times \sum_{j=1}^{2} q_{i j}(k)+I_{b_{1}}(k)+I_{v_{i}}(k)-\Delta D_{b}(k)$

$\min f_{2}\left(g(k), x_{o s}\right)=\sum_{i=1}^{2}\left(\sum_{j=1}^{2} h_{s_{i}}(k) \times \sum_{j=1}^{2} q_{i j}(k)+I_{v_{i}}(k)\right)$

S.t.

$\left\{\begin{array}{l}\Delta g_{i j}^{\prime}(k) \leq \Delta g_{i j}^{\prime \max } \\ \Delta g_{i j}(k) \leq \Delta g_{i j}^{\text {max }} \\ 0 \leq x_{o s 1} \leq T \\ 0 \leq x_{\text {os2 }} \leq T\end{array}\right.$

\section{Simulation test}

Three types of vehicle delays, including the general vehicle (car) delay, bus delay, and the entire intersection vehicle delay, were used to measure the performance of different experiments. Fig.5 shows the average vehicle delays and stops for buses and general vehicles respectively. In terms of the overall benefit of $\mathrm{Bi}$-objective Optimization Model adaptive signal control, the figures show that, when compared with the NEMA fully actuated signal control, the Bi-objective Optimization Model adaptive signal control consistently resulted in significant delay improvement for the entire intersection. .It reduced the intersection delay by $14.7 \%$ and stops by $23.2 \%$ for that fixed timing parameters.

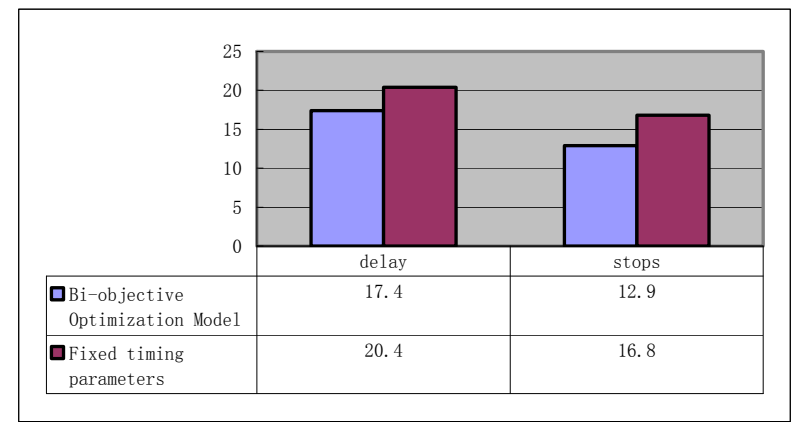

Figure 5. Contrasting delay and stops for two signal timing scheme

\section{CONCLUSIONS}

TSP is a useful tool for the efficiency of easing traffic jams. However, total vehicle queue delay and stops in non-prioritized phases is rarely being considered simultaneously in the previous model. This paper presented a bi-objective optimization model. It reduces total vehicle queue delay and stops including the prioritized and non-prioritized phases. Then an analytical example for the design and evaluation of a transit signal priority system with Matlab and Vissim.The result shows that the proposed method is promising in that it considers stops in non-prioritized phases. The analytical approach can be used as a supplementary tool to TSP.

\section{REFERENCES}

[1] Guangwei Zhou,Albert Gan,L.David Shen. Optimization of Adaptive Transit Signal Priority Using Parallel Genetic Algorithm[J].Tsinghua Science And Technology, 2007, 12 (2) : 131-140.

[2] Ma Wanjing,Cheng Xuebao,Wu Zhizhou,Yang Xiaoguang. A coordinated intersection-group bus signal priority control approach 
[J]. CHINA CIVIL ENGINEERING JOURNAL 2009 , 42(2):105-111

[3] WonhoKim.An Improved Transit Signal Priority System for Networks with $\quad$ Nearside BusStops[C].Washington.D.C.:Transportation Researeh Board 84th Annual Meeting, 2004.

[4] Liu H. " Development of Adaptive Transit Signal Priority Systems B, Theoretical Approach”. Institute of transportation Studies, University of California, Berkeley, 2005.

[5] Sunkari S R, Beasley P S, Urbanik T, et al. "Model to evaluate the impacts of bus priority on signalized intersections". Transportation Research Record 1494, Transportation Research Board, Washington, D.C., 1995,pp. 117-123.

[6] Lin G S, Liang P, Schonfeld P. Adaptive control of transit operations[C]. Maryland:University of Maryland, 1995.

[7] Jinwoo Lee, Amer Shalaby.Advanced Transit Signal Priority Contro1 Using on- line Microsimulation -based Transit Prediction Model[C].washingtonD.C.: Transportation Researeh Board 83rd Annual Meeting, 2003.

[8] P1tuMirehandani, LaryHead, AnnaKnyazyan. An Approaeh Towards the Intergration of Bus Priority and Traffiec Adaptive Signal Control[C].T WashingtonD.C.:Transportation Research Board 80th Annual Meeting, 2000.

[9] Gang-len Chang, Meenakshy Vasudevan, Chih-chiang Su. Modeling and evaluation of adaptive bus-preemption control with and without automatic vehicle location systems [J].Transportation Research A,1996,30(4):251-268.

[10] Dion F, Rakha H, Zhang Y. "Evaluation of potential transit signal priority benefits along a fixed-time signalized arterial". Transp. Eng., vol,130(3),2004,pp.294-303. 